\title{
Whole genome resequencing data for rock pigeon (Columba livia)
}

\author{
Ali Esmailizadeh ${ }^{1,4}$, Hamed Kharrati-Koopaee ${ }^{1,2^{*}}$ (D) and Hojjat Assadoullahpour Nanaei ${ }^{3}$
}

\begin{abstract}
Objective: Navigation is the most important feature of homing pigeons, however no integrated response to genetic mechanism of navigation has been reported. The generated data herein represent whole-genome resequencing data for homing pigeon and three other breeds of rock pigeons. Selective sweep analysis between homing pigeon and other breeds of rock pigeon can provide new insight about identification of candidate genes and biological pathways for homing pigeon ability.

Data description: Whole-genomes sequence data related to 95 birds from four breeds of rock pigeons including, 29 feral pigeons, 24 Shiraz tumblers, 24 Persian high flyers and 18 homing pigeons were provided. More than 6.94 billion short reads with coverage (average $\approx 7.50 \mathrm{x}$ ) and $407.1 \mathrm{~Gb}$ data were produced. Whole genome sequencing was carried out on the Illumina Hiseq 2000 platform using a 350 bp library size and 150 bp paired-end read lengths. The whole genome sequencing data have been submitted th the NCBI SRA Database (PRJNA532675). The presented data set can provide useful genomic information to explain the genetic mechanism of navigation ability of homing pigeons and also testing other genetic hypothesis by genomic analysis.
\end{abstract}

Keywords: Rock pigeon, Navigation, Genome analysis

\section{Objective}

Domestic pigeon (Columba livia domestica) is a frequent bird around the world that was derived from the rock pigeon. They are native to Europe, North Africa, the Middle East, and South Asia. [1]. C. livia is known as an ideal model for different investigation such as ecology, genetics, physiology, behavior, and anatomical diversity [2]. The geographic origins of pigeon breeds have not been well described. However, it has been demonstrated that the origins of major breed groups of pigeons go back to the Middle East and North America [3]. Like other domestic animals, natural and artificial selections have remarkably impacted the genetic evolution in pigeons [4].

\footnotetext{
*Correspondence: h.kharrati.ko@gmail.com

${ }^{1}$ Department of Animal Science, Faculty of Agriculture, Shahid Bahonar

University of Kerman, PB 76169-133, Kerman, Iran

Full list of author information is available at the end of the article
}

More than 350 breeds of pigeons have been reported and about 300 species of pigeons from family Columbidae are kept as pet [1]. For several reasons such as food source, decoration and fly sporting, pigeons have been kept for a long time. However, navigation is the most important feature of pigeons. Homing pigeon (also called racing homer) is able to navigate for finding the home's way. This ability of pigeons played a vital role in wars to transfer the news of wars. Several investigations have been attempted to explain the mechanism of navigation in pigeons, but it has been remained as a puzzle $[1,3]$. At present, several hypotheses have been proposed for the mechanisms of navigation ability in domestic pigeons, the most important of them are summarized in the following statements. The sun (solar compass) [5], the earth's magnetic (magnetic compass) [6], the olfactory and visual pathways $[7,8]$ and the specialized development of the hippocampus in brain [3]. Among these

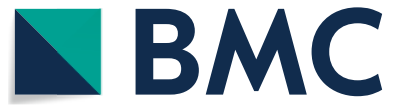

(c) The Author(s) 2021. Open Access This article is licensed under a Creative Commons Attribution 4.0 International License, which permits use, sharing, adaptation, distribution and reproduction in any medium or format, as long as you give appropriate credit to the original author(s) and the source, provide a link to the Creative Commons licence, and indicate if changes were made. The images or other third party material in this article are included in the article's Creative Commons licence, unless indicated otherwise in a credit line to the material. If material is not included in the article's Creative Commons licence and your intended use is not permitted by statutory regulation or exceeds the permitted use, you will need to obtain permission directly from the copyright holder. To view a copy of this licence, visit http://creativecommons.org/licenses/by/4.0/. The Creative Commons Public Domain Dedication waiver (http://creativeco mmons.org/publicdomain/zero/1.0/) applies to the data made available in this article, unless otherwise stated in a credit line to the data. 
theories, the orientation of pigeons using the earth's magnetic field, called magnetoreception, is more considered as an environmental factor [9]. It also should be noted that hippocampus contribute to navigation in homing pigeons. Hippocampus is one of complex brain structure and has a critical role in learning and memory [10]. We carried out selective sweep analysis by generating 95 whole genomes sequence of different breeds of pigeons and several candidate genes and biological pathways were reported for homing pigeon ability [10]. The presented data set can provide interesting and applied resources to understand the genetic mechanism of navigation ability of homing pigeons and also examination of other associated hypothesis by genomic analysis.

\section{Data description}

For selective sweep analysis, we collected 95 blood samples of rock pigeon breeds, including 29 feral pigeons, 24. Shiraz tumblers, 24 Persian high flyers and 18 homing pigeons. In this way, more than 6.94 billion short reads with coverage (average $\approx 7.50 \mathrm{x}$ ) and $407.1 \mathrm{~Gb}$ data were generated [10] (Table 1). The collected rock pigeon's breeds show different ability in navigation. As an example homing pigeon and feral pigeon have the strongest and weakest navigation ability and also Persian high flyer and Shiraz tumblers have the same navigation ability. The samples were obtained from four regions (Shiraz, Tehran, Kerman, and Marvdasht) in Iran. DNA was provided by phenol-chlorroform protocol. Agarose gel (1\%) and absorption ratio 260/280

Table 1 Overview of whole-genome sequence data files of four breeds of rock pigeons

\begin{tabular}{|c|c|c|c|}
\hline Label & Name of data file/data set & $\begin{array}{l}\text { File type (file } \\
\text { extension) }\end{array}$ & Data repository and identifier (DOI or accession number) \\
\hline Bioproject & Columba livia Genome sequencing & Fastq (fq.gz) & https://identifiers.org/ncbi/bioproject:PRJNA532675 [13] \\
\hline Data set 1 & s76_KIZ-FER1/ Feral rock pigeon & Fastq (fq.gz) & $\begin{array}{l}\text { NCBI SRA Database } \\
\text { https://identifiers.org/ncbi/insdc.sra:SRR10102989 [14] }\end{array}$ \\
\hline Data set 1 & s107_KIZ-FER29/ Feral rock pigeon & & $\begin{array}{l}\text { NCBI SRA Database } \\
\text { https://identifiers.org/ncbi/insdc.sra:SRR10102958 [15] }\end{array}$ \\
\hline Data set 1 & s86_KIZ-FER10/ Feral rock pigeon & & $\begin{array}{l}\text { NCBI SRA Database } \\
\text { https://identifiers.org/ncbi/insdc.sra:SRR10102979 [16] }\end{array}$ \\
\hline Data set 1 & s92_KIZ-FER16/ Feral rock pigeon & & $\begin{array}{l}\text { NCBI SRA Database } \\
\text { https://identifiers.org/ncbi/insdc.sra:SRR10102972 [17] }\end{array}$ \\
\hline Data set 2 & s26_KIZ-HOP1/Homing Pigeon & Fastq (fq.gz) & $\begin{array}{l}\text { NCBI SRA Database } \\
\text { https://identifiers.org/ncbi/insdc.sra:SRR10103035 [18] }\end{array}$ \\
\hline Data set 2 & s37_KIZ-HOP10/ Homing Pigeon & & $\begin{array}{l}\text { NCBI SRA Database } \\
\text { https://identifiers.org/ncbi/insdc.sra:SRR10103025 [19] }\end{array}$ \\
\hline Data set 2 & s47_KIZ-HOP17/ Homing Pigeon & & $\begin{array}{l}\text { NCBI SRA Database } \\
\text { https://identifiers.org/ncbi/insdc.sra:SRR10103017 [20] }\end{array}$ \\
\hline Data set 2 & s48_KIZ-HOP18/ Homing Pigeon & & $\begin{array}{l}\text { NCBI SRA Database } \\
\text { https://identifiers.org/ncbi/insdc.sra:SRR10103016 [21] }\end{array}$ \\
\hline Data set 3 & s1_KIZ-PHF1/ Persian high flyer & Fastq (fq.gz) & $\begin{array}{l}\text { NCBI SRA Database } \\
\text { https://identifiers.org/ncbi/insdc.sra:SRR10103052 [22] }\end{array}$ \\
\hline Data set 3 & s8_KIZ-PHF8/ Persian high flyer & & $\begin{array}{l}\text { NCBI SRA Database } \\
\text { https://identifiers.org/ncbi/insdc.sra:SRR10102985 [23] }\end{array}$ \\
\hline Data set 3 & s23_KIZ-PHF22/ Persian high flyer & & $\begin{array}{l}\text { NCBI SRA Database } \\
\text { https://identifiers.org/ncbi/insdc.sra:SRR10103038 [24] }\end{array}$ \\
\hline Data set 3 & s25_KIZ-PHF24/ Persian high flyer & & $\begin{array}{l}\text { NCBI SRA Database } \\
\text { https://identifiers.org/ncbi/insdc.sra:SRR10103036 [25] }\end{array}$ \\
\hline Data set 4 & s51_KIZ-SHT1/ Shiraz tumbler & Fastq (fq.gz) & $\begin{array}{l}\text { NCBI SRA Database } \\
\text { https://identifiers.org/ncbi/insdc.sra:SRR10103015 [26] }\end{array}$ \\
\hline Data set 4 & s66_KIZ-SHT16/Shiraz tumbler & & $\begin{array}{l}\text { NCBI SRA Database } \\
\text { https://identifiers.org/ncbi/insdc.sra:SRR10102999 [27] }\end{array}$ \\
\hline Data set 4 & s68_KIZ-SHT18/ Shiraz tumbler & & $\begin{array}{l}\text { NCBI SRA Database } \\
\text { https://identifiers.org/ncbi/insdc.sra:SRR10102997 [28] }\end{array}$ \\
\hline Data set 4 & s74_KIZ-SHT24/ Shiraz tumbler & & $\begin{array}{l}\text { NCBI SRA Database } \\
\text { https://identifiers.org/ncbi/insdc.sra:SRR10102990 [29] }\end{array}$ \\
\hline
\end{tabular}

The whole genome sequencing data was submitted at NCBI SRA Database with accession number PRJNA532675. A total 95 whole genome sequence files were generated for four different breeds of rock pigeons including feral rock pigeons ( 29 samples), homing pigeon (18 samples), Persian high flyer ( 24 samples) and Shiraz tumbler (24 samples). This Table shows the link for the bioproject and also four links were showed for each breeds of rock pigeons 
(nm) were applied to evaluation of the extracted DNA. Library size $350 \mathrm{bp}$ and paired-end short read length 150 bp were generated by Illumina Hiseq 2000 [10]. Btrim (version: 2.0) was used to adaptors trimming and quality control of short reads. Clean short reads were aligned by default parameters of BWA-MEM (http:// bio-bwa.sourceforge.net) against the pigeon reference genome (assembly accession: GCF_000337935.1) [10, 11]. SortSam and MarkDuplicates in Picard-tools-1.56 (http://broadinstitute.github.io/picard), was utilized to sort bam files and mark duplicates. SAMtools program (v0.1.19-44428cd) was applied to index the mapping files. Local realignment and base quality recalibration were performed by GATK (v2.6-4-g3e5ff60). The UnifiedGenotyper scripts of GATK was utilized for SNPs detection, and also SNPs filtration was carried out by the VariantFiltration in GATK. Annotation of SNPs were reported by SnpEff software (v4.3T) [10]. Around 20.6 million single nucleotide polymorphisms (SNPs) were reported after quality filtering of the detected SNPs, with $\sim 7.6 \times$ average sequence depth for each individual. In order to identify the associated selective sweep with homing ability between racing homer pigeon and residual breeds of pigeon, the genomewide distributions of fixation index (Fst) was estimated based on the previous explained method [12]. The outcomes of signature selection analysis showed GSR gene (encoding glutathione-disulfide reductase) might be considered as result of positive selection in the homing pigeon. Gene expression analysis uncovered that GSR was highly expressed in the wattle and visual pigment cell layer, and displays increased expression levels in the homing pigeon. Our finding provides new insight about importance of the hippocampus for homing ability, and the potential role of GSR in pigeon magnetoreception.

\section{Limitations}

The number of the breeds of rock pigeon collected and their genomes sequenced is a limitation of our investigation. We could only obtain four breeds for whole genome sequencing. In addition, it should be noted that, we produced the short-reads with a mean coverage of about $7.50 \times$ and probably, the data set could not support some genomic analyses.

\section{Abbreviations \\ Gb: Giga byte; NCBI: The National Center for Biotechnology Information; BWA: Burrows-Wheeler aligner; SNP: Single nucleotide polymorphism; GATK: Genomic analysis toolkit; bp: Base pair; DNA: Deoxyribonucleic acid.}

\section{Acknowledgements}

The authors appreciate the pigeon breeders in Iran for their assistance in collecting pigeon samples.

\section{Authors' contributions}

AE conceived the study. Sampling was done by HAN. The genome resequencing data were created and assessed by AE and HKK. HKK prepared the manuscript. All authors read and approved the final manuscript.

\section{Funding}

This work was supported by the National Natural Science Foundation of China (31822048) and Strategic Priority Research Program of the Chinese Academy of Sciences (XDB13020600), and the Animal Branch of the Germplasm Bank of Wild Species of Chinese Academy of Science.

\section{Availability of data and materials}

The whole genome sequence data described herein have been deposited in NCBI database as the sequence read archive (SRA) format (https://www. ncbi.nlm.nih.gov/sra/?term=PRJNA532675) under the accession number of PRJNA532675. Please see Table 1 and the references [13-29] for details and links to the data.

\section{Declarations}

\section{Ethics approval and consent to participate}

The procedure of blood sampling was approved by the Department of Animal Science at Shiraz University (Permit number: 93-192).

\section{Consent for publication}

Not applicable.

\section{Competing interests}

The authors declare that they have no competing interests.

\section{Author details}

${ }^{1}$ Department of Animal Science, Faculty of Agriculture, Shahid Bahonar University of Kerman, PB 76169-133, Kerman, Iran. ${ }^{2}$ Institute of Biotechnology, Shiraz University, Shiraz, Iran. ${ }^{3}$ Key Laboratory of Animal Genetics, Breeding and Reproduction of Shaanxi Province, College of Animal Science and Technology, Northwest A\&F University, Yangling 712100, China. ${ }^{4}$ State Key Laboratory of Genetic Resources and Evolution, Kunming Institute of Zoology, Chinese Academy of Sciences, No. 32 Jiaochang Donglu, Kunming 650223, Yunnan, China.

Received: 4 May 2021 Accepted: 27 July 2021

Published online: 09 August 2021

\section{References}

1. Shapiro MD, Domyan ET. Domestic pigeons. Curr Biol. 2013;23(8):R302-3.

2. Holt C, Campbell M, Keays DA, Edelman N, Kapusta A, Maclary E, Domyan E, Suh A, Warren WC, Yandell M, Gilbert MT. Improved genome assembly and annotation for the rock pigeon (Columba livia). G3 Genes Genom Genet. 2018;8(5):1391-8.

3. Shapiro MD, Kronenberg Z, Li C, Domyan ET, Pan H, Campbell M, Tan H, Huff CD, Hu H, Vickrey Al, Nielsen SC. Genomic diversity and evolution of the head crest in the rock pigeon. Science. 2013;339(6123):1063-7.

4. Gregory TR. Artificial selection and domestication: modern lessons from Darwin's enduring analogy. Evol Ed Outre. 2009;2(1):5-27.

5. Muheim R, Moore FR, Phillips JB. Calibration of magnetic and celestial compass cues in migratory birds-a review of cue-conflict experiments. J Exp Biol. 2006;209(1):2-17.

6. Whitlock JR, Heynen AJ, Shuler MG, Bear MF. Learning induces long-term potentiation in the hippocampus. Science. 2006;313(5790):1093-7.

7. Biro D, Meade J, Guilford T. Familiar route loyalty implies visual pilotage in the homing pigeon. P Natl Acad Sci. 2004;101(50):17440-3.

8. Rehkämper G, Frahm HD, Cnotka J. Mosaic evolution and adaptive brain component alteration under domestication seen on the background of evolutionary theory. Brain Behav Evol. 2008;2:115-26.

9. Wiltschko W, Wiltschko R. Magnetic orientation and magnetoreception in birds and other animals. J Comp Physiol A. 2005;191 (8):675-93.

10. Shao Y, Tian HY, Zhang JJ, Kharrati-Koopaee H, Guo X, Zhuang XL, Li ML, Nanaie HA, Dehghani Tafti E, Shojaei B, Reza NM. Genomic and 
phenotypic analyses reveal mechanisms underlying homing ability in pigeon. Mol Biol Evol. 2020;37(1):134-48.

11. Li H, Durbin R. Fast and accurate short read alignment with BurrowsWheeler transform. Bioinformatics. 2009;25(14):1754-60.

12. Shriver MD, Kennedy GC, Parra EJ, Lawson HA, Sonpar V, Huang J, Akey $\mathrm{JM}$, Jones KW. The genomic distribution of population substructure in four populations using 8,525 autosomal SNPs. Hum Genomics. 2004; 1(4):1-3.

13. NCBI SRA Database. https://identifiers.org/ncbi/bioproject:PRJNA532675. (2020).

14. NCBI SRA Database. https://identifiers.org/ncbi/insdc.sra:SRR10102989. (2020).

15. NCBI SRA Database. https://identifiers.org/ncbi/insdc.sra:SRR10102958. (2020).

16. NCBI SRA Database. https://identifiers.org/ncbi/insdc.sra:SRR10102979. (2020).

17. NCBI SRA Database. https://identifiers.org/ncbi/insdc.sra:SRR10102972. (2020)

18. NCBI SRA Database. https://identifiers.org/ncbi/insdc.sra:SRR10103035. (2020).

19. NCBI SRA Database. https://identifiers.org/ncbi/insdc.sra:SRR10103025. (2020).

20. NCBI SRA Database. https://identifiers.org/ncbi/insdc.sra:SRR10103017. (2020).
21. NCBI SRA Database. https://identifiers.org/ncbi/insdc.sra:SRR10103016. (2020).

22. NCBI SRA Database. https://identifiers.org/ncbi/insdc.sra:SRR10103052 (2020).

23. NCBI SRA Database. https://identifiers.org/ncbi/insdc.sra:SRR10102985. (2020).

24. NCBI SRA Database. https://identifiers.org/ncbi/insdc.sra:SRR10103038. (2020).

25. NCBI SRA Database. https://identifiers.org/ncbi/insdc.sra:SRR10103036. (2020).

26. NCBI SRA Database. https://identifiers.org/ncbi/insdc.sra:SRR10103015. (2020).

27. NCBI SRA Database. https://identifiers.org/ncbi/insdc.sra:SRR10102999. (2020).

28. NCBI SRA Database. https://identifiers.org/ncbi/insdc.sra:SRR10102997. (2020).

29. NCBI SRA Database. https://identifiers.org/ncbi/insdc.sra:SRR10102990. (2020).

\section{Publisher's Note}

Springer Nature remains neutral with regard to jurisdictional claims in published maps and institutional affiliations.
Ready to submit your research? Choose BMC and benefit from:

- fast, convenient online submission

- thorough peer review by experienced researchers in your field

- rapid publication on acceptance

- support for research data, including large and complex data types

- gold Open Access which fosters wider collaboration and increased citations

- maximum visibility for your research: over $100 \mathrm{M}$ website views per year

At BMC, research is always in progress.

Learn more biomedcentral.com/submissions 